\title{
$\operatorname{ARCH}(p)$ 模型的严平稳遍历性和高阶矩*
}

\author{
陈 敏 安鸿志 \\ (中国科学院应用数学研究所, 北京 100080)
}

\section{关键词 $\mathrm{ARCH}(p)$ 模型 严平稳遍历 高阶矩}

考虑如下非线性随机递推方程:

$$
\left\{\begin{array}{l}
x_{t}=\varepsilon_{t} h_{t}^{1 / 2}, \\
h_{t}=\alpha_{0}+\alpha_{1} x_{t-1}^{2}+\cdots+\alpha_{p} x_{t-p}^{2},
\end{array}\right.
$$

其中 $\alpha_{0}>0, \alpha_{i} \geqslant 0, i=1,2, \cdots, p,\left\{\varepsilon_{t}\right\}$ 是 i.i.d 随机序列, $E \varepsilon_{t}=0, E \varepsilon_{t}^{2}=1$. 称模型 (1) 为 $\operatorname{ARCH}(p)$ (Autoregressive conditional heteroscedasticity) 模型. 它由 Engle ${ }^{[1]}$ 首先引人并被广泛地用于 计量经济建设, 如通货膨胀率、兑换率、利率和股票价值 (应用文献见 J. of Econometrics, 52 (1992)，1 311 及其所引文献). 但关于模型 (1) 的严平稳遍历性和高阶矩存在的条件, 至今 尚无满意的结果, 而这些条件是时间序列统计推断的基础. Nelson ${ }^{[2]}$ 对 ARCH(1) 给出了严平. 稳遍历的充要条件, 他的结果无法推广到 $\mathrm{ARCH}(p)$ 的情形. Bougeral 和 Picard ${ }^{[3]}$ 给出了 $\operatorname{ARCH}(p)$ 存在严平稳遍历解的充要条件, 但他们在同一篇文章中又指出这一条件的验证是非 常困难的, 只能通过 Monte Carlo 方法来模拟估计. 而关于高阶矩存在的条件仅有 ARCH(1) 的结果. 因此, 当 $p \geqslant 2$ 时, 对 $\mathrm{ARCH}(p)$ 模型参数估计渐近理论往往是基于严平稳遍历解和 高阶矩存在的前提下来讨论的 ${ }^{[4]}$. 本文的目的是在较一般的条件下给出模型 (1) 有严平稳遍 历解的充要条件和高阶矩存在的条件. 利用这些结果, 我们在另文中获得了 $\operatorname{ARCH}(p)$ 模 型具有 $\operatorname{ARCH}(p)$ 误差的回归或自回归模型参数估计的强相合性和渐近正态性.

设随机序列 $\left\{x_{t}\right\}$ 服从随机递推方程 (1). 令

$$
y_{t}^{2}=x_{t}^{2}, \eta_{t}=\varepsilon_{t}^{2}, Y_{t}=\left(y_{t}, \cdots, y_{t-p+1}\right)^{z}, B_{t}=\left(\alpha_{0} \eta_{t}, 0, \cdots, 0\right)^{\tau},
$$

$$
A_{t}=\left(\begin{array}{cccc}
\alpha_{1} \eta_{t} & \cdots & \alpha_{p-1} \eta_{t} & \alpha_{p} \eta_{t} \\
1 & \cdots & 0 & 0 \\
\vdots & \ddots & \vdots & \vdots \\
0 & \cdots & 1 & 0
\end{array}\right), \quad A=E A_{t}=\left(\begin{array}{cccc}
\alpha_{1} E \eta_{t} & \cdots & \alpha_{p-1} E \eta_{t} & \alpha_{p} E \eta_{t} \\
1 & \cdots & 0 & 0 \\
\vdots & \ddots & \vdots & \vdots \\
0 & \cdots & 1 & 0
\end{array}\right)=\left(\begin{array}{cccc}
\alpha_{1} & \cdots & \alpha_{p-1} & \alpha_{p} \\
1 & \cdots & 0 & 0 \\
\vdots & \ddots & \vdots & \vdots \\
0 & \cdots & 1 & 0
\end{array}\right)
$$

由(1) 式有

即

$$
x_{t}^{2}=\varepsilon_{t}^{2}\left(\alpha_{0}+\alpha_{1} x_{t-1}^{2}+\cdots+\alpha_{p} x_{t-p}^{2}\right)=\alpha_{1} \varepsilon_{t}^{2} x_{t-1}^{2}+\cdots+\alpha_{p} \varepsilon_{t}^{2} x_{t-p}^{2}+\alpha_{0} \varepsilon_{t}^{2}
$$

$$
y_{t}=\alpha_{1} \eta_{t} y_{t-1}+\cdots+\alpha_{p} \eta_{t} y_{t-p}+\alpha_{0} \eta_{t}
$$

1994-11-10 收稿

*国家自然科学基金利中国科学院应用数学研究所概率青年实验室资助项目 
因此, $y_{t}$ 是 (3) 的解当且仅当 $Y_{\mathrm{t}}$ 是如下随机差分方程的解:

$$
Y_{t}=A_{t} Y_{t-1}+B_{t} \text {. }
$$

引理 1 若 $\sum_{j=1}^{p} \alpha_{j}<1$, 则随机级数 $\sum_{k=1}^{\infty}\left(\prod_{j=0}^{k-1} A_{t-j}\right) B_{t-k}$, a.s. 收玫. 进一步, 若

$$
Y_{t}=B_{t}+\sum_{k=1}^{\infty}\left(\prod_{j=0}^{k-1} A_{t-j}\right) B_{t-k}
$$

则 $\left\{Y_{t}\right\}$ 是严平稳遍历的随机向量序列, 且服从 (4) 式.

证 由 $A_{t}$ 和 $B_{t}$ 的定义可见, $\left\{A_{t}\right\}$ 和 $\left\{B_{t}\right\}$ 是两个独立同分布的非负随机矩阵 (向量) 序列, 且当 $k \neq j$ 时, $A_{t-j}$ 与 $B_{t-k}$ 独立. 因此

$$
E\left(\prod_{j=0}^{k-1} A_{t-j}\right) B_{t-k}=\left(\prod_{j=0}^{k-1} E A_{t-j}\right) E B_{t-k}=A^{k}\left(\alpha_{0}, 0, \cdots, 0\right)^{\tau} .
$$

容易验证, $A$ 的谱半径 $\rho(A)<1$ 当且仅当 $\sum_{j=1}^{p} \alpha_{j}<1$. 这样, 若 $\sum_{j=1}^{p} \alpha_{j}<1$, 则 $\sum_{k=1}^{\infty} A^{k}<\infty^{[5]}$. 这就意味 着 $\sum_{k=1}^{\infty} E\left(\prod_{j=0}^{k-1} A_{t-j}\right) B_{t-k}<\infty$, 故 $\sum_{k=1}^{\infty}\left(\prod_{j_{x}=0}^{k-1} A_{t-j}\right) B_{t-k}<\infty$, a.s..

由文献[6], 容易验证由 (5) 式定义的随机向量序列 $\left\{Y_{t}\right\}$ 是严平稳遍历的. 且有

$$
Y_{\mathrm{t}}=B_{\mathrm{t}}+A_{t}\left[B_{t-1}+\sum_{k=2}^{\infty}\left(\prod_{j=1}^{k-1} A_{t-j}\right) B_{t-k}\right]=B_{t}+A_{t} Y_{t-1} .
$$

引理 2 若随机序列 $\left\{Y_{t}\right\}$ 是模型 (1) 的严平稳解, 且 $E Y_{0}<\infty$, 则 $\sum_{j=1}^{p} \alpha_{j}<1$. 进一步, 若随机 序列 $\left\{U_{t}\right\}$ 是模型 (1) 的另一严平稳解, 且 $E U_{0}<\infty$, 则 $U_{t}=Y_{t}$, a.s..

证 由(4)式有

$$
Y_{0}=A_{0} Y_{-1}+B_{0}=\cdots=B_{0}+\sum_{k=1}^{n-1}\left(\prod_{j=0}^{k-1} A_{-j}\right) B_{-k}+\left(\prod_{j=0}^{n-1} A_{-j}\right) Y_{-n},
$$

注意到 $A_{n}, B_{n}$ 和 $Y_{n}$ 都是非负的, $\left\{A_{n}\right\}$ 是独立同分布序列, 且对 $i \neq j, A_{n-j}$ 与 $B_{n-i}$ 独立, 因此

$$
E Y_{0} \geqslant \sum_{k=1}^{n-1} E\left(\prod_{j=0}^{k-1} A_{-j}\right) B_{-k}=\sum_{k=1}^{n-1} A^{k} B
$$

其中 $B=\left(\alpha_{0}, 0, \cdots, 0\right)^{x}$, 这就意味着 $\sum_{k=1}^{\infty} A^{k} B<\infty$, 故

$$
\lim _{n \rightarrow \infty} A^{n} B=0 .
$$

令 $\delta_{i}=(0, \cdots, 0,1,0, \cdots, 0)^{\tau}, 1 \leqslant i \leqslant p$, 如果能证明对 $1 \leqslant i \leqslant p$,

$$
\lim _{n \rightarrow \infty} A^{n} \delta_{i}=0 \text {, }
$$

则有 $\lim _{n \rightarrow \infty} A^{n}=0$, 这就意味着 $\rho(A)<1^{[9]}$, 而这等价于 $\sum_{j=1}^{p} \alpha_{j}<1$. 事实上, 由 $B=\alpha_{0} \delta_{1}$ 和 (7) 式知, 对 
$i=1,(8)$ 式成立. 又由 $A \delta_{p}=\alpha_{p} \delta_{1}$, 有

$$
\lim _{n \rightarrow \infty} A^{n} \delta_{p}=\alpha_{p} \lim _{n \rightarrow \infty} A^{n-1} \delta_{1}=0 .
$$

如果对某一 $2<i \leqslant p,(8)$ 式成立, 则由于 $A \delta_{i-1}=\alpha_{i-1} \delta_{1}+\delta_{i}$, 有

$$
\lim _{n \rightarrow \infty} A^{n} \delta_{i-1}=\lim _{n \rightarrow \infty} A^{n-1}\left(\alpha_{i-1} \delta_{1}+\delta_{i}\right)=0 \text {. }
$$

因此, 由归纳法知, 对任意 $1 \leqslant i \leqslant p$,(8) 式成立.

下证唯一性. 令 $V_{t}=Y_{t}-U_{t}$, 则

$$
V_{t}=Y_{t}-U_{t}=\left(\prod_{j=0}^{t-1} A_{t-j}\right)\left(Y_{0}-U_{0}\right) .
$$

为方便, 我们表矩阵 $C$ 的 $(i, j)$ 元为 $(C)_{i j}$, 下证对 $1 \leqslant i \leqslant p$, 存在常数 $c>0$ 和 $0<\lambda<1$ 使得

$$
E\left|\left[\left(\prod_{j=0}^{t-1} A_{t-j}\right)\left(Y_{0}-U_{0}\right)\right]_{i}\right| \leqslant c \lambda^{t}
$$

这样由 Borel-Cantelli 引理就有 $\lim _{n \rightarrow \infty} V_{t}=0$, a.s..

由于证法类似, 我们仅对 $i=1$ 给出 (9) 式的证明. 由 $\left\{A_{t}\right\}$ 的独立性, 易见 $E\left[\left(\prod_{j=0}^{t-1} A_{t \cdot j}\right)_{\text {Is }}\right]=$ $\left(A^{t}\right)_{s s^{.}}$注意到 $\rho(A)<1$, 存在常数 $\varepsilon>0$, 满足 $\rho(A)+\varepsilon<1$ 和常数 $M>0$, 使得

$$
\left(A^{t}\right)_{1 \mathrm{~s}} \leqslant M[\rho(A)+\varepsilon]^{t[5]} \text {. }
$$

由 (5) 式知, $Y_{0}, U_{0}$ 是 $S_{0}=\sigma\left(A_{s}, B_{s}, s \leqslant 0\right)$ 可测的, 这样由 $\left\{A_{t}\right\}$ 的独立性有

$$
\begin{gathered}
E\left|\left[\left(\prod_{j=0}^{t-1} A_{t-j}\right)\left(Y_{0}-U_{0}\right)\right]_{l}\right|=E\left|\sum_{k=1}^{p}\left(\prod_{j=0}^{t-1} A_{t-j}\right)_{t s}\left(Y_{0}-U_{0}\right)_{s}\right| \leqslant \\
\sum_{k=1}^{p} E\left[\left(\prod_{j=0}^{t-1} A_{t-j}\right)_{I s}\right] E\left|\left(Y_{0}-U_{0}\right)_{s}\right| \leqslant C[\rho(A)+\varepsilon]^{t} .
\end{gathered}
$$

定理 $1\left\{x_{t}\right\}$ 是 $\operatorname{ARCH}(p)$ 模型 (1) 的严平稳解, 且 $E x_{0}^{2}<\infty$ 的充要条件是 $\sum_{j=1}^{p} \alpha_{j}<1$. 此外, 这个严平稳解还是遍历的、唯一的.

证 设 $\left\{x_{t}\right\}$ 是模型 (1) 的严平稳解, 且 $E x_{0}^{2}<\infty$, 由 (2) 和 (3) 式知 $Y_{t}$ 是 (4) 式的严平稳解, 且 $E Y_{0}<\infty$, 这样由引理 2 知 $\sum_{j=1}^{p} \alpha_{j}<1$. 反之, 若 $\sum_{j=1}^{p} \alpha_{j}<1$, 由引理 1 知, $\left\{Y_{t}\right\}$ 是 (4) 式的严平稳遍 历解. 再由 (2) (4) 式知 $\left\{x_{t}^{2}\right\}$ 是严平稳遍历的. 由 (1) 式 $x_{t}=\varepsilon_{t}\left(\alpha_{0}+\alpha_{1} x_{t-1}^{2}+\cdots+\alpha_{p} x_{t}^{2}\right)^{1 / 2}$, 知 $\left\{x_{t}\right\}$ 是严平稳遍历的.

唯一性由引理 2 即得.

推论 $1\left\{x_{t}\right\}$ 是 $\operatorname{ARCH}(p)$ 模型 (1) 的宽平稳解的充要条件是 $\sum_{j=1}^{p} \alpha_{j}<1$.

以上已证, 当 $\sum_{j=1}^{p} \alpha_{j}<1$ 时, (1) 式有严平稳遍历解. 且 


$$
x_{t}^{2}=\delta_{1}^{\tau}\left[B_{t}+\sum_{k=1}^{\infty}\left(\prod_{j=0}^{k-1} A_{t-j}\right) B_{t-k}\right] .
$$

记 $L^{r}=\left\{\left.x\left|\|x\|_{r}=E^{1 / r}\right| x\right|^{r}<\infty, x\right.$ 是随机变量 $\}$, 对矩阵 $C, C^{\otimes_{m}}$ 表 $m$ 个 $C$ 的 Kronecker 积, $\operatorname{Vec}(C)$ 表 $C$ 的拉直运算.

定理 2 设 $\left\{x_{t}\right\}$ 服从 $\operatorname{ARCH}(p)$ 模型 $(1), E \varepsilon_{t}^{4}<\infty, \sum_{j=1}^{p} \alpha_{j}<1$, 记 $\Sigma_{2}=E\left(A_{t}^{\otimes 2}\right)$, 则当 $\rho\left(\Sigma_{2}\right)<1$ 时, $x_{t}^{2} \in L^{2}$.

证 令 $S_{n}(t)=B_{t}+\sum_{k=1}^{n}\left(\prod_{j=0}^{k-1} A_{t-j}\right) B_{t-k}$,

则容易验证

$$
W_{n+m, n}(t)=S_{n+m}(t)-S_{n}(t), m \geqslant 1 \text { 任意, }
$$

$$
W_{n+m, n}(t)=A_{t} W_{n-1+m, n-1}(t-1) .
$$

再记 $\Gamma_{n+m, n}^{2}(t)=\operatorname{Vec}\left(W_{n+m, n}(t) W_{n+m, n}^{\tau}(t)\right)$, 由矩阵 Kronecker 积和拉直运算的性质及 (11) 式, 容 易验证

$$
\begin{aligned}
\Gamma_{n+m, n}^{2}(t)= & \operatorname{Vec}\left(A_{t} W_{n-1+m, n-1}(t-1) W_{n-1+m, n-1}^{\tau}(t-1) A_{t}^{\tau}\right)= \\
& \left(A_{t}^{\otimes 2}\right) \operatorname{Vec}\left(W_{n-1+m, n-1}(t) W_{n-1+m, n-1}^{\tau}(t-1)\right)=\left(A_{t}^{\otimes 2}\right) \Gamma_{n-1+m, n-1}^{2}(t-1) .
\end{aligned}
$$

由 $\Gamma_{n-1+m, n-1}^{2}(t-1)$ 和 $W_{n-1+m, n-1}(t-1)$ 的定义知, $\Gamma_{n-1+m, n-1}^{2}(t-1)$ 是 $\mathscr{Y}_{t-1}=\sigma\left(A_{s}, B_{s}, \mathrm{~s} \leqslant \mathrm{t}-1\right)$ 可 测的. 再由 $A_{t}$ 独立同分布, 由 (12) 式有

而

$$
\begin{gathered}
E \Gamma_{n+m, n}^{2}(t)=E\left(A_{t}^{\otimes 2}\right) E \Gamma_{n-1+m, n-1}^{2}(t-1)=\left[E\left(A_{t}^{\otimes 2}\right)\right]^{2} E \Gamma_{n-2+m, n-2}^{2}(t-2)= \\
\cdots=\left[E\left(A_{t}^{\otimes 2}\right)\right]^{n} E \Gamma_{m, 0}^{2}(t-n), \\
\Gamma_{m, 0}^{2}(t-n)=\operatorname{Vec}\left(W_{m, 0}(t-n) W_{m, 0}^{\tau}(t-n)\right),
\end{gathered}
$$

$$
W_{m, 0}(t-n)=S_{m}(t-n)-S_{0}(t-n)=\sum_{k=1}^{m}\left(\prod_{j=0}^{k-1} A_{t-n-j}\right) B_{t-n-k} \underline{=}\left(w_{1}, \cdots, w_{p}\right)^{\tau},
$$

这里 $w_{i}=\sum_{k=1}^{m} \alpha_{0}\left(\prod_{j=0}^{k-1} A_{t-n-j}\right)_{i 1} \varepsilon_{t-n-k}^{2}$. 因此, $\Gamma_{m, 0}^{2}(t-n)$ 中的元素形如 $w_{i}^{s_{1}} w_{j}^{s_{2}}, s_{1}, s_{2}=0,1, \cdots, p$, $s_{1}+s_{2}=2, i, j=1,2, \cdots, p$, 于是由 Hölder 和 Minkowski 不等式, 并注意到

$$
\begin{aligned}
{\left[\left(\prod_{j=0}^{k-1} A_{t-n-j}\right)_{i 1}\right]^{2}=} & {\left[\left(\prod_{j=0}^{k-1} A_{t-n-j}\right) \otimes\left(\prod_{j=0}^{k-1} A_{t-n-j}\right)\right]_{i 1, i 1}=} \\
& {\left[\sum_{j=0}^{k-1}\left(A_{t-n-j} \otimes A_{t-n-j}\right)\right]_{i 1, i 1}, }
\end{aligned}
$$

其中第一二等号后的 $(\cdot)_{i 1, i 1}$ 表示原来元素在 Kronecker 积中的位置, 类似于 (9) 式的证法有

$$
\begin{aligned}
\left(E w_{i}^{2}\right)^{1 / 2}= & \left\{E\left[\sum_{k=1}^{m} \alpha_{0}\left(\prod_{j=0}^{k-1} A_{t-n-j}\right)_{i 1} \varepsilon_{t-n-k}^{2}\right]^{2}\right\}^{1 / 2} \leqslant \\
& \sum_{k=1}^{m} \alpha_{0}\left\{E\left[\left(\prod_{j=0}^{k-1} A_{t-n-j}\right)_{i 1}\right]^{2}\right\}^{1 / 2}\left(E \varepsilon_{t-n-k}^{4}\right)^{1 / 2} \leqslant
\end{aligned}
$$




$$
\begin{aligned}
& c \sum_{k=1}^{m}\left\{\sum_{j=0}^{k-1} E\left[\left(A_{t-n-j} \otimes A_{t-n-j}\right)\right]_{i 1, i 1}\right\}^{1 / 2} \leqslant \\
& c \sum_{k=1}^{m}\left\{\left[\left(E\left(A_{t}^{\otimes 2}\right)\right)^{k}\right]_{i 1, i 1}\right\}^{1 / 2} \leqslant c \sum_{k=1}^{m} \lambda^{k / 2} .
\end{aligned}
$$

记 $\mu_{2 m}=\max _{1 \leqslant i \leqslant p^{2}}\left\{\left(E \Gamma_{m, 0}^{2}(t-n)\right)_{i}\right\}$, 则由 (14) 式, 当 $m \rightarrow \infty$ 有 $\mu_{2 m}<\infty$, 从而由 (13) 式有

$$
E\left[\Gamma_{n+m . n}^{2}(t)\right]_{1} \leqslant\left(c p^{2} \mu_{2 m}\right) \lambda^{n} .
$$

这里及 (14) 式中 $c$ 为常数, 其含意不总相同, 又类似于 (12) 式证法有

$$
\begin{aligned}
{\left[\delta_{1}^{\tau} W_{n+m, n}(t)\right]^{2}=} & \delta_{1}^{\tau} W_{n+m, n}(t) W_{n+m, n}^{\tau}(t) \delta_{1}=\operatorname{Vec}\left(\delta_{1}^{\tau} W_{n+m, n}(t) W_{n+m, n}^{\tau}(t) \delta_{1}\right)= \\
& \left(\delta_{1}^{\otimes 2}\right)^{\tau} \operatorname{Vec}\left(W_{n+m, n}(t) W_{n+m, n}^{\tau}(t)\right)=\left[\Gamma_{n+m, n}^{2}(t)\right]_{1} .
\end{aligned}
$$

于是由 (14) 和 (15) 式, 并注意到 $0<\lambda<1$, 便有

$$
\begin{gathered}
\left\|\delta_{1}^{\tau} W_{n+m, n}(t)\right\|_{2}=\left\{E\left[\delta_{1}^{\tau} W_{n+m, n}(t)\right]^{2}\right\}^{1 / 2}=\left\{E\left[\left(\Gamma_{n+m, n}^{2}(t)\right)_{1}\right]^{2}\right\}^{1 / 2} \leqslant \\
c \mu_{2 m} \lambda^{\frac{n}{2}} \rightarrow 0(n \rightarrow \infty, m \rightarrow \infty) .
\end{gathered}
$$

此刞 $x_{t, n}^{2}=\delta_{i}^{\tau}\left[B_{t}+\sum_{k=1}^{n}\left(\prod_{j=0}^{k-1} A_{t-j}\right) B_{t-k}\right]$ 是 $L^{2}$ 中的 Cauchy 基本列, 故 $x_{t}^{2} \in L^{2}$.

定理 3 设 $\left\{x_{t}\right\}$ 服从 $\mathrm{ARCH}(p)$ 模型 $(1), E \varepsilon_{t}^{4(r-1)}<\infty, r>2$ 是整数, $\sum_{i=1}^{p} \alpha_{j}<1$, 记 $\Sigma_{r}=$ $E\left(A_{t}^{\otimes r}\right)$, 则当 $\rho\left(\Sigma_{r}\right)<1$ 时, $x_{t}^{2} \in L^{r}$.

证 记 $S_{n}(t), W_{n+m, n}(t)$ 如定理 2. 令

$$
\Gamma_{n+m, n}^{r}(t)=\operatorname{Vec}\left(\cdots \operatorname{Vec}\left(\operatorname{Vec}\left(W_{n+m, n}(t) W_{n+m, n}^{\tau}(t)\right) W_{n+m, n}^{\tau}(t)\right) \cdots \cdots W_{n+m, n}^{\tau}(t)\right),
$$

类似于 (12) 式的证法, 容易验证

$$
\Gamma_{n+m, n}^{r}(t)=\left(A_{t}^{\otimes r}\right) \Gamma_{n-1+m, n}^{r} \quad(t-1) .
$$

从湍有

$$
E \Gamma_{n+m, n}^{r}(t)=\left[E\left(A_{t}^{\otimes r}\right)\right] E \Gamma_{n-1+m, n-1}^{r}(t)=\cdots=\left[E\left(A_{t}^{\otimes r}\right)\right]^{n} E \Gamma_{m, 0}^{r}(t-n) .
$$

用类似丁定理 2 的证法知, $\Gamma_{m, 0}^{r}(t-n)$ 中元素形如

$$
w_{1}^{s_{1}} w_{2}^{s_{2}} \cdots w_{p}^{s_{p}}, s_{1}, \cdots, s_{p}=0,1, \cdots, p, s_{1}+\cdots+s_{p}=r
$$

H. $\mu_{r m}=\max _{i \leqslant i \leqslant p^{\prime}}\left\{\left(E \Gamma_{m, 0}^{r}(t-n)\right)_{i}\right\}<\infty(m \rightarrow \infty)$. 又

$$
\begin{aligned}
{\left[\delta_{1}^{\tau} W_{n+m, n}(t)\right]^{r}=} & \delta_{1}^{\tau} W_{n+m, n}(t) W_{n+m, n}^{\tau}(t) \delta_{1} W_{n+m, n}^{\tau}(t) \delta_{1} \cdot \cdots \cdot W_{n+m, n}^{\tau}(t) \delta_{1}= \\
& \operatorname{Vec}\left(\delta_{1}^{\tau} W_{n+m, n}(t) W_{n+m, n}^{\tau}(t) \delta_{1}\right) W_{n+m, n}^{\tau}(t) \delta_{1} \cdot \cdots \cdot W_{n+m, n}^{\tau}(t) \delta_{1}= \\
& \left(\delta_{1}^{\otimes 2}\right)^{\tau} \operatorname{Vec}\left(W_{n+m, n}(t) W_{n+m, n}^{\tau}(t)\right) W_{n+m, n}^{\tau}(t) \delta_{1} \cdot \cdots \cdot W_{n+m, n}^{\tau}(t) \delta_{1}= \\
& \left(\delta_{1}^{\otimes 3}\right)^{\tau} \operatorname{Vec}\left(\operatorname{Vec}\left(W_{n+m, n}(t) W_{n+m, n}^{\tau}(t)\right) W_{n+m, n}^{\tau}(t)\right) W_{n+m, n}^{\tau}(t) \delta_{1} \cdot \cdots \cdot W_{n+m, n}^{\tau}(t) \delta_{1}= \\
& \cdots=\left(\delta_{1}^{\otimes r}\right)^{\tau} \Gamma_{n+m, n}^{r}(t)=\left(\Gamma_{n+m, n}^{r}(t)\right)_{1} .
\end{aligned}
$$


于是由 (16)，(17)式有

$$
\begin{gathered}
\left\|\delta_{1}^{\tau} W_{n+m, n}(t)\right\|_{r}=\left\{E\left[\delta_{1}^{\tau} W_{n+m, n}(t)\right]^{r}\right\}^{1 / r}=\left\{E\left[\left(\Gamma_{n+m, n}^{r}(t)\right)_{1}\right]^{r}\right\}^{1 / r} \leqslant \\
c \mu_{r m} \lambda^{n / r} \rightarrow 0(n \rightarrow \infty, m \rightarrow \infty) .
\end{gathered}
$$

因此 $x_{t, n}^{2}=\delta_{1}^{\tau}\left[B_{t}+\sum_{k=1}^{n}\left(\prod_{j=0}^{k-1} A_{t-j}\right) B_{t-k}\right]$ 是 $L^{r}$ 中的 Cauchy 基本列, 故 $x_{t}^{2} \in L^{r}$.

\section{参 考 文 献}

1 Engle R F. Autoregressive conditional heteroskedasticity with estimates of the variance of $\mathrm{U} K$ inflation. Econometrica, 1982, 50:987 1008

2 Nelson D B. Stationarity and persistence in $\operatorname{GARCH}(1,1)$ model. Econometric Theory, 1990, 6:318 334

3 Bougeral P, Picard N. Stationarity of GARCH processes and some nonnegative time series. J Econometrics, 1992, 52: $115 \sim 127$

4 Weiss A A. Asymptotic theory for ARCH models: estimation and testing. Econometric Theory, 1986, 2:107 131.

5 Horn R A, Johnson C R. Matrix Analysis. Cambridge: Cambridge Univ Press, 1985. 290 311

6 王梓坤著. 随机过程论. 北京: 科学出版社, 1978. 290 306 